\title{
Redundant miR-3077-5p and miR-705 mediate the shift of mesenchymal stem cell lineage commitment to adipocyte in osteoporosis bone marrow
}

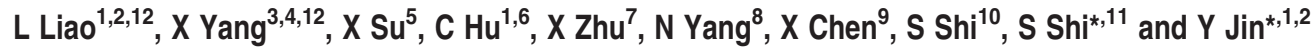

During the process of aging, especially for postmenopausal females, the cell lineage commitment of mesenchymal stem cells (MSCs) shift to adipocyte in bone marrow, resulting in osteoporosis. However, the cell-intrinsic mechanism of this cell lineage commitment switch is poorly understood. As the post-transcription regulation by microRNAs (miRNAs) has a critical role in MSCs differentiation and bone homeostasis, we performed comprehensive miRNAs profiling and found miR-705 and miR-3077$5 p$ were significantly enhanced in MSCs from osteoporosis bone marrow. Both miR-705 and miR-3077-5p acted as inhibitors of MSCs osteoblast differentiation and promoters of adipocyte differentiation, by targeting on the $3^{\prime}$ untranslated region ( $3^{\prime}$ UTR) of HOXA10 and RUNX2 mRNA separately. Combined inhibition of miR-705 and miR-3077-5p rescued the cell lineage commitment disorder of MSCs through restoring HOXA10 and RUNX2 protein level. Furthermore, we found excessive TNF $\alpha$ and reactive oxygen species caused by estrogen deficiency led to the upregulation of both miRNAs through NF- $\kappa$ B pathway. In conclusion, our findings showed that redundant miR-705 and miR-3077-5p synergistically mediated the shift of MSCs cell lineage commitment to adipocyte in osteoporosis bone marrow, providing new insight into the etiology of osteoporosis at the posttranscriptional level. Moreover, the rescue of MSCs lineage commitment disorder by regulating miRNAs expression suggested a novel potential therapeutic target for osteoporosis as well as stem cell-mediated regenerative medicine.

Cell Death and Disease (2013) 4, e600; doi:10.1038/cddis.2013.130; published online 18 April 2013

Subject Category: Cancer

Osteoporosis is a bone metabolism disease characterized by a systemic impairment of bone mass and increase of bone marrow fat, which results in increased propensity of fragility fractures. ${ }^{1-4}$ This disturbance of homeostasis between bone and bone marrow fat are widely considered to be caused by the shift of cell lineage commitment of mesenchymal stem cells (MSCs) to adipocyte. ${ }^{4-8}$ Downregulation of hormones and cytokines, such as estrogen, insulin-like growth factor1 (IGF1), transforming growth factor beta1 (TGF $\beta 1$ ) and interleukin11 (IL-11), had been suggested to result in the MSCs lineage commitment switch. ${ }^{9,10}$ However, the cell-intrinsic mechanism is still poorly understood.

The final cell fate decision of MSCs relies on the activation of lineage-specific transcription factors and repression of transcription factors promoting commitment to other lineages. ${ }^{11-14}$ For example, osteoblastic differentiation of MSCs is controlled by Runx2, Osterix and DIx5, whereas $\mathrm{PPAR} \gamma$ and $\mathrm{C} / \mathrm{EBP} \alpha$ are the master transcription factors in adipocyte commitment. ${ }^{15}$ However, in a transcription factor profiling, the mRNAs of the majority of the transcription factors that regulate MSCs differentiation were not altered in MSCs derived from aged bone. ${ }^{7}$ Our preliminary gene expression profiling of MSCs derived from osteoporosis bone marrow showed a similar result, suggesting a limitation of investigating the cell-intrinsic mechanism of osteoporosis merely at the transcription level.

Recently, researches about microRNAs (miRNAs) provided direct implications for fundamental biology as well as disease etiology and treatment. ${ }^{16}$ As the factor for post-transcription

\footnotetext{
${ }^{1}$ Research and Development Center for Tissue Engineering, Fourth Military Medical University, Xi'an, China; ${ }^{2}$ Department of Oral Histology and Pathology, School of Stomatology, Fourth Military Medical University, Xi'an, China; ${ }^{3}$ Department of Prosthetics, School of Stomatology, Fourth Military Medical University, Xi'an, China; ${ }^{4}$ Department of Prosthetics, School of Stomatology, ZunYi Medical College, ZunYi, China; ${ }^{5}$ Department of Orthodontics, Stomatology Hospital of Xi'an Jiaotong University, Xi'an, China; ${ }^{6}$ Engineering Technology Center for Tissue Engineering of Xi'an, Xi'an, China; ${ }^{7}$ Department of Pediatric Dentistry, Yantai Stomatology Hospital, Yantai, China; ${ }^{8}$ Department of Stomatology, the PLA 309th Hospital, Beijing, China; ' ${ }^{9}$ Department of Orthodontics, Affiliated Stomatology Hospital, Medical College of Zhejiang University, HangZhou, China; ${ }^{10}$ Center for Craniofacial Molecular Biology, Ostrow School of Dentistry, University of Southern California, Los Angeles, CA, USA and ${ }^{11}$ Department of Stomatology, 306 Hospital of PLA, Beijing, China

*Corresponding author: Y Jin, Research and Development Center for Tissue Engineering, Fourth Military Medical University, 145 West Changle Road, Xi'an 710032 , China. Tel: + 8629 84776147; Fax: + 8629 83218039; E-mail: yanjin@fmmu.edu.cn

Or S Shi, Department of Stomatology, 306 Hospital of PLA, No 9 Anxiang Beili, Beijing 100101, China. Tel: +86 1066356729 ; Fax: +86 10 64876056; E-mail: shishengen@sohu.com

${ }^{12}$ These authors contributed equally to this work.

Keywords: mesenchymal stem cells; microRNA; osteoporosis; cell differentiation

Abbreviations: MSCs, mesenchymal stem cells; miRNA, microRNA; miR, microRNA; UTR, untranslated regions; RUNX2, runt-related transcription factor 2; HOX, homeobox gene; LPL, lipoprotein lipase; PPAR $\gamma$, peroxisome proliferator-activated receptor gamma; OCN, osteocalcin; ROS, reactive oxygen species; NF- $\kappa$ B, nuclear factor of kappa light polypeptide gene enhancer in B-cells; OVX, ovariectomy; Sham, sham surgery

Received 11.12.12; revised 21.2.13; accepted 15.3.13; Edited by M Federici
} 
regulation, emerging evidences showed miRNAs are crucial for physiological bone development and MSCs differentiation. ${ }^{17}$ A cluster of miRNAs were reported to target the $3^{\prime}$ untranslated region ( $3^{\prime} U T R$ ) of the mRNA of lineage-specific genes, such as RUNX2, PPAR $\gamma, \operatorname{HOXA10}$, and so on. ${ }^{17}$ Therefore, maintaining the proper level of miRNAs is crucial for MSCs lineage commitment. However, whether disrupted miRNAs expression is related with bone disease is largely unknown. Recently, two groups showed that the mutation in pre-miRNA or polymorphisms in miRNA target sites were closely related with primary osteoporosis. ${ }^{18} \mathrm{~A}$ miRNA profiling in MSCs from aged rhesus macaque found aberrant microRNAs expression with advanced age. ${ }^{19}$ Moreover, our recent work found that pathological condition (inflammation) inhibited osteogenic differentiation of MSCs through disturbing miRNAs expression, ${ }^{20}$ suggesting aberrant miRNAs expression is an unexplored cell-intrinsic mechanism of MSCs differentiation disorder during osteoporosis.
In this study, we performed comprehensive miRNAs profiling, and found miR-705 and miR-3077-5p were significantly enhanced in osteoporotic MSCs. As both miRNAs were certified as inhibitor of osteogenic differentiation and promoter of adipogenic differentiation of MSCs, their enhancement synergistically mediated the shift of MSCs lineage commitment to adipocyte. Furthermore, excessive TNF $\alpha$ and reactive oxygen species (ROS) caused by estrogen deficiency led to the upregulation of both miRNAs through NF- $\kappa$ B pathway. Importantly, the knockdown of miR-705 and miR-3077-5p rescued the differentiation disorder of osteoporotic MSCs, suggesting a potential therapeutic target for osteoporosis as well as regenerative medicine.

\section{Results}

Cell lineage commitment of MSCs shifts to adipocyte during osteoporosis. To confirm bone loss of ovariectomised a
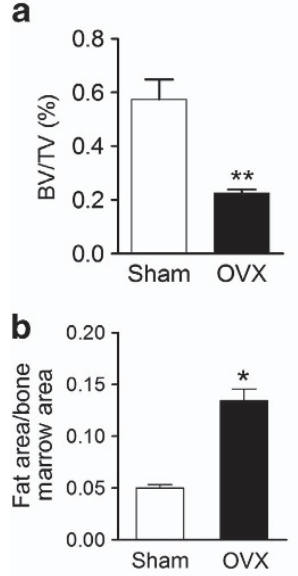

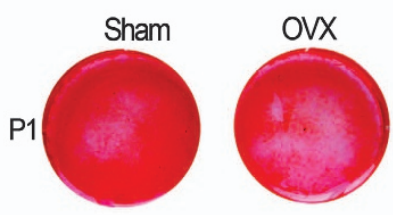

e

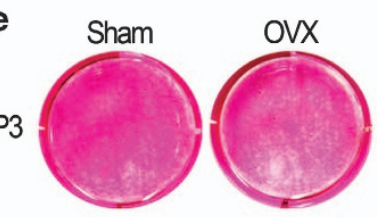

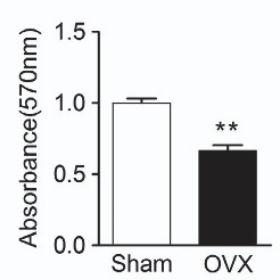

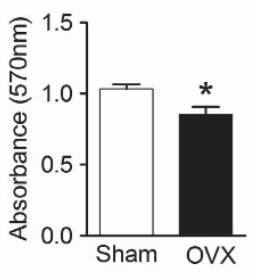

d

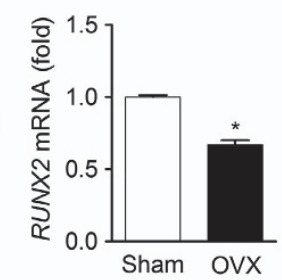

f

P3

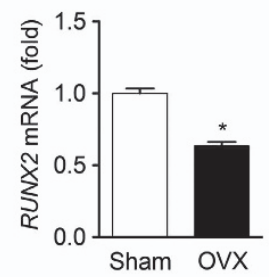

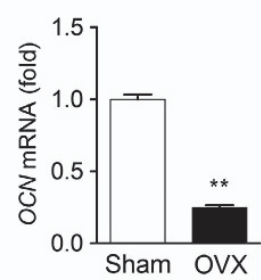

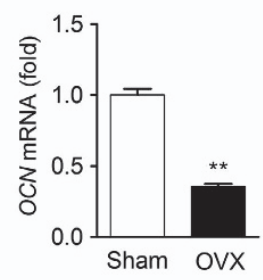

g
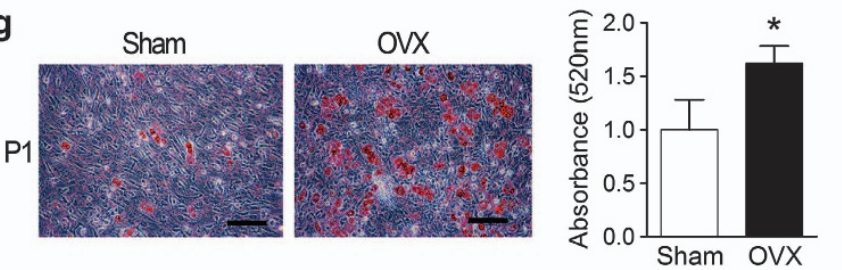

i

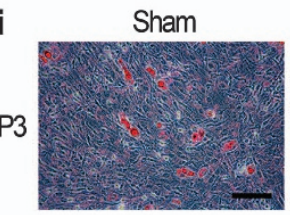

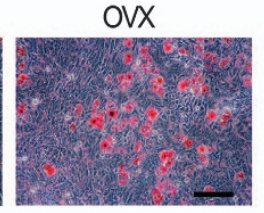

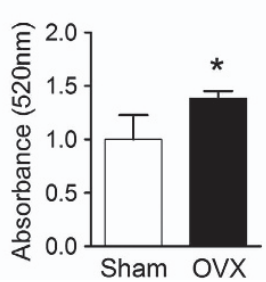

h
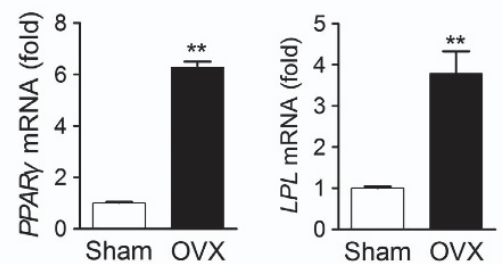

$\mathbf{j}$
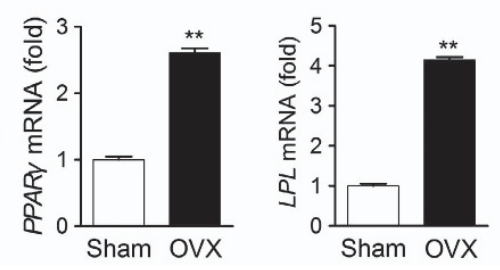

Figure 1 Shift of cell lineage commitment in MSCs from osteoporosis bone marrow. (a) The femurs from Sham and OVX mice were scanned by Micro-CT 3 months after surgery. The structural parameter of the trabecular bone volume (BV/TV: \%) was obtained. (b) The bone marrow fat was detected by toluidine blue staining and quantified with Image-Pro software. (c-f) Impaired osteoblast differentiation in MSCs from osteoporosis bone marrow. First passage (P1) and third passage (P3) MSCs from OVX and sham surgery bone marrow were induced with osteogenic medium for 14 days. Alizarin red staining was performed and quantified via extraction with cetylpyridinium chloride in P1 MSCs (c) and P3 MSCs (e). The expression of RUNX2 and OCN in P1 MSCs (d) and P3 MSCs (f) was measured by real-time RT-PCR and were shown as fold induction relative to Sham. (g-j) Enhanced adipocyte differentiation in MSCs from osteoporosis bone marrow. Oil red 0 staining was performed after 7 days of adipogenic induction in P1 MSCs (g) and P3 MSCs (i). The representative microscopic view at a magnification of $\times 200$ of cells after staining was shown. The oil red 0 staining was quantified via extraction with isopropanol. The expression of PPARy and LPL was measured by real-time RT-PCR in P1 MSCs (h) and P3 MSCs (j). Data are shown as means \pm S.D. ${ }^{\star} P<0.05,{ }^{* \star} P<0.01$ versus Sham, $n=3$. Scale bar in micrographs represents $50 \mu \mathrm{m}$. Sham, sham surgery; OVX, ovariectomy; P1, first passage; P3, third passage; BV/TV, bone volume/tissue volume 
mice, we performed micro-CT analysis 3 months after ovariectomy (OVX). The results showed that the trabecular bone structure was destroyed, and bone mass density (BMD) was significantly reduced in OVX femurs (Supplementary Figures S1A and B). Morphological indices of trabecular bone were all decreased, demonstrating obvious bone loss (Figure 1a, Supplementary Figures S1C and D). In contrast, histological assay showed that the fat amount was significantly increased in OVX bone marrow (Figure 1b, Supplementary Figure S1F). Although the body weight (Supplementary Figure S1E) and bone marrow fat amount were increased, the size of adipocytes in OVX bone marrow was not changed (Supplementary Figure S1G).

To investigate the characteristics of osteoporotic MSCs, we isolated MSCs from bone marrows of OVX and Sham mice and performed analysis at the first passage. Flow-cytometry analysis showed that both OVX and Sham MSCs highly expressed SCA-1, CD29, CD73, CD105 and CD106, while they did not express CD34 and CD45 (Supplementary Figures S2A and B). Proliferation assay showed no significant difference between OVX and Sham MSCs (data not shown). However, we found that OVX MSCs formed less-mineralized nodules after osteogenic induction (Figure 1c). Real-time reverse transcription $\mathrm{PCR}$ showed that osteogenic marker RUNX2 and OCN mRNA were significantly decreased in OVX MSCs (Figure 1d). In contrast, adipocyte formation and adipogenic marker PPAR $\gamma$ and LPL mRNA were enhanced in OVX BMSCs after adipogenic induction (Figures $1 \mathrm{~g}$ and $\mathrm{h}$ ). To further confirm a cell-intrinsic defect in MSCs, we repeated the differentiation assay using the third passage MSCs. As expected, the osteogenic differentiation was inhibited (Figures 1e and f), while the adipogenic differentiation was promoted in the third passage OVX MSCs (Figures 1i and $j$ ).

miR-705 and miR-3077-5p overexpression in MSCs from osteoporosis bone marrow. To investigate the miRNAs expression in osteoporotic MSCs, we performed comprehensive miRNAs profiling in OVX and Sham MSCs using miRNA microarray. Among 1040 mouse miRNAs registered in miRBase database (Release 17.0, www.mirbase.org), 339 miRNAs were detected in MSCs. Statistical analysis showed the expression of 10 miRNAs were different between OVX and Sham MSCs (Figure 2a). Among them, the difference of miR-705 and miR-3077-5p were most significant between the two groups. Real-time RT-PCR confirmed the enhancement of miR-705 and miR-3077-5p in OVX MSCs (Figure 2b). Notably, their expression level in OVX MSCs remained higher than Sham MSCs at the third passage (Figure 2c). To further confirm the relevance between miR-705/miR-3077 and osteoporosis, we treated the osteoporosis mice by $17 \beta$ estradiol (E2) for 4 weeks and detected the miRNAs. Being used as pharmacological agents to prevent postmenopausal bone loss, E2 injection significantly recovered the trabecular bone number and volume in femurs of OVX mice (data not shown). Consistent with the recovery of osteoporosis, we found that E2 treatment significantly reduced excessive miR-705 and miR-3077-5p in OVX mice (Figure 2d).

To verify the tissue-specificity of miR-705 and miR-3077$5 p$, we profiled their expression in different tissues. The expression of miR-705 and miR-3077-5p in bone were higher than in other tissues (Figure 2e). Moreover, the microarray chip signal intensity (SI) of miR-705 $(\mathrm{SI}=8292)$ and miR$3077-5 p(S I=4392)$ were ranked among the top $10 \%$ of 339 miRNA detected in MSCs, much higher than the average SI $(\mathrm{SI}=1234)$ and median $\mathrm{SI}(\mathrm{SI}=59)$.

Inhibition of MSCs osteoblast lineage commitment by miR-705 and miR-3077-5p. To investigate whether miR705 and miR-3077-5p have a role in MSCs osteoblast lineage commitment, we tested their expression level at different time points of osteogenic differentiation. The results showed that the expression levels of both miR-705 and miR-3077-5p decreased significantly following osteogenic a
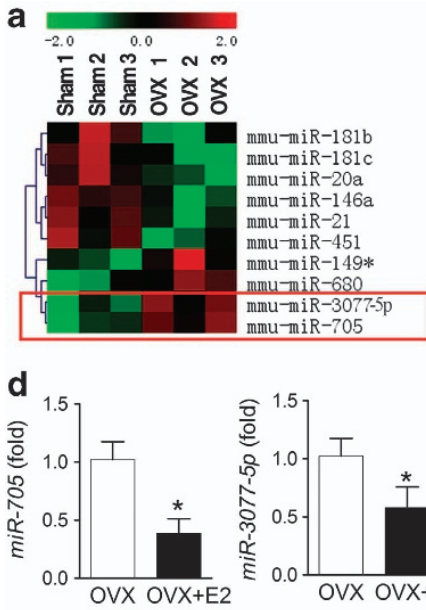

b
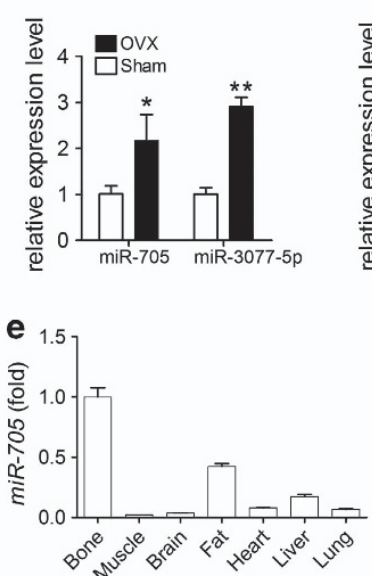

C
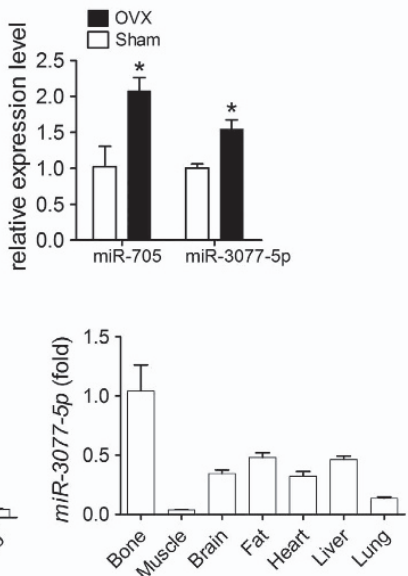

Figure 2 miR-705 and miR-3077-5p are enhanced in MSCs from osteoporosis bone marrow. (a) The heat map of miRNAs differentially expressed between Sham and OVX MSCs. (b, c) Levels of miR-705 and miR-3077-5p in first passage (b) and third passage (c) MSCs were determined using real-time RT-PCR. (d) OVX mice were treated with estradiol for 4 weeks and real-time RT-PCR analysis were performed to determine miR-705 and miR-3077-5p expression level. (e) Real-time RT-PCR analysis of miR-705 and miR-3077-5p expression in various mouse tissues. Data are shown as means \pm S.D. ${ }^{*} P<0.05$ versus Sham, $n=3$. Sham, sham surgery; OVX, ovariectomy; E2, estradiol injection 
differentiation and reached the lowest level on the 7th day, then slightly increased on the 10th day (Figures $3 a$ and b).

To confirm their biological function in osteoblast lineage commitment, we overexpressed or knocked down miR-705 and miR-3077-5p expression in MSCs by specific synthetic miRNA mimics (mimics of mature miRNA) or inhibitors (complementary to mature miRNA) (Supplementary Figures S3A and B). After 14 days of osteogenic induction, real-time RT-PCR showed that the effects of miRNA mimics and inhibitor lasted during induction (Supplementary Figure $\mathrm{S} 4 \mathrm{~A}$ ). Alizarin red staining showed that overexpression of miR-705 or miR-3077-5p significantly reduced the mineralized node formation, whereas their knockdown them promoted mineralization of MSCs (Figures $3 \mathrm{c}$ and e). Consistently, the expression of osteogenic marker RUNX2 and OCN were suppressed by overexpression of miR-705 or miR-3077-5p, whereas it was promoted by their knockdown (Figures $3 d$ and f).
Promotion of MSCs adipocyte lineage commitment by miR-705 and miR-3077-5p. To examine whether miR-705 and miR-3077-5p also have an important role in the adipocyte differentiation of MSCs, we tested their expression at different time points of adipogenic differentiation. Real-time RT-PCR showed the expression of miR-705 or miR-3077-5p to be significantly increased since the first day and reached the highest level on the 7th day, then slightly decreased from the top after 10 days of induction (Figures $4 a$ and $b$ ).

Then, we performed gain or loss of function assay to assess their function in MSCs adipogenic differentiation (Supplementary Figure S4B). The results showed overexpression of miR-705 or miR-3077-5p significantly increased adipocyte formation from MSCs after 7 days of adipogenic induction. Consistently, the knockdown of both miRNAs reduced adipocyte formation (Figures $4 \mathrm{c}$ and e). Real-time RT-PCR showed that the mRNAs of adipogenic marker
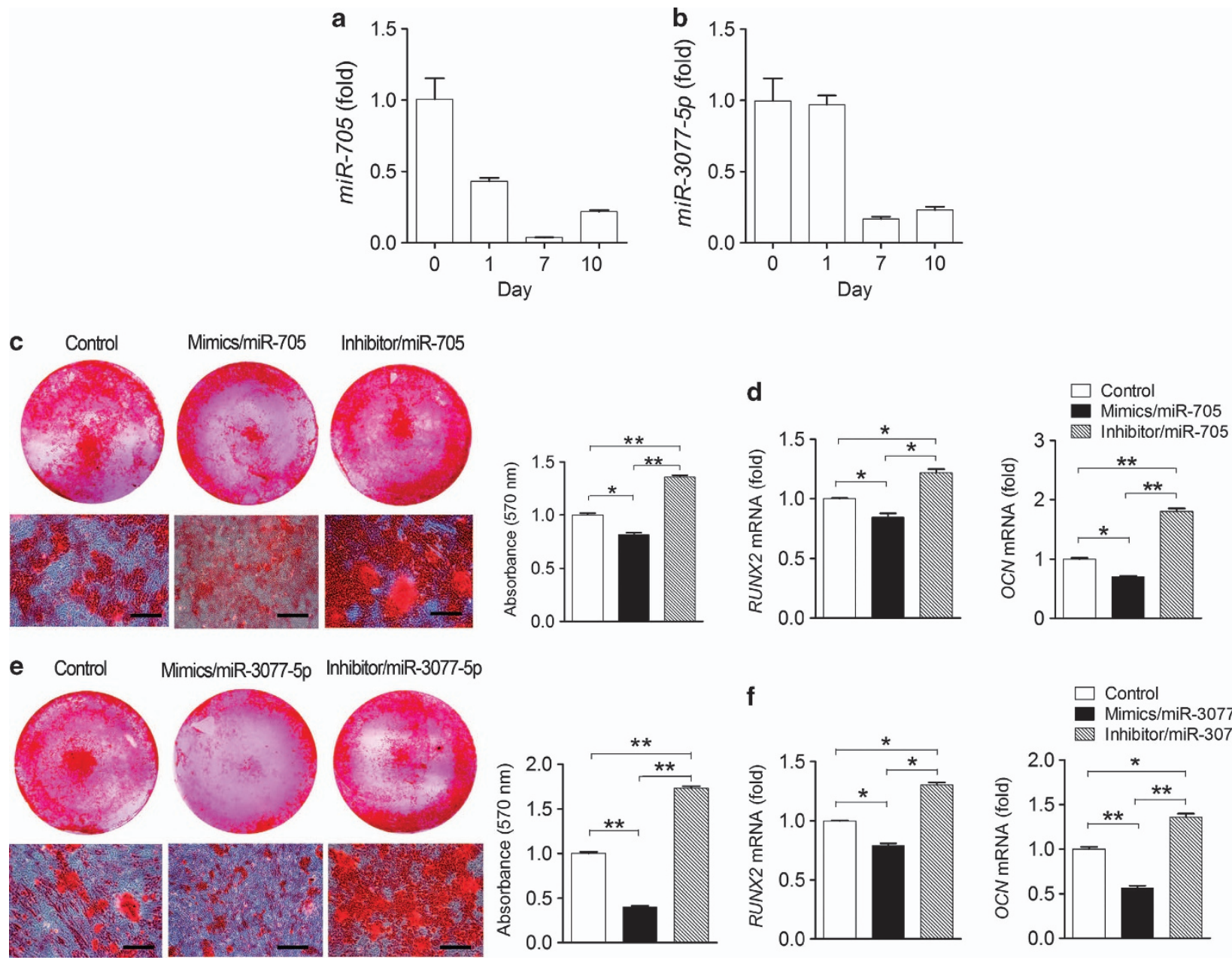

Figure 3 miR-705 and miR-3077-5p Inhibit osteoblast differentiation of MSCs. ( $\mathbf{a}$ and $\mathbf{b}$ ) Real-time RT-PCR was used to determine the expression level of miR-705 and miR-3077-5p during different time point of osteogenic induction. (c and d) miR-705 inhibited osteoblast differentiation of MSCs. miR-705 mimics, inhibitors and negative controls were transfected into MSCs. Two days after transfection, MSCs were induced with osteogenic medium for 14 days. Alizarin red staining was performed and quantified (c). The representative microscopic view at a magnification of $\times 100$ of cells after staining was shown. The expression of RUNX2 and OCN mRNA were measured by realtime RT-PCR (d). (e and f) miR-3077-5p inhibited osteoblast differentiation of MSCs. miR-3077-5p mimics, inhibitors and negative controls were transfected into MSCs and induced with osteogenic medium for 14 days. Alizarin red staining was performed and quantified (e). The expression of RUNX2 and OCN mRNA were measured by real-time RT-PCR (f). Data are shown as means \pm S.D. ${ }^{*} P<0.05,{ }^{\star \star} P<0.01, n=3$. Scale bar in micrographs represents $100 \mu \mathrm{m}$ 

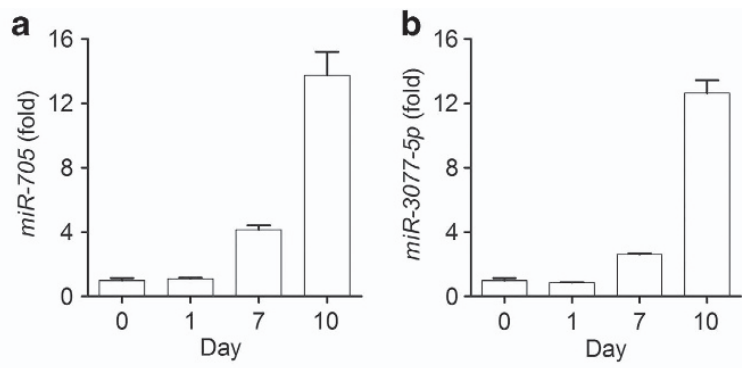
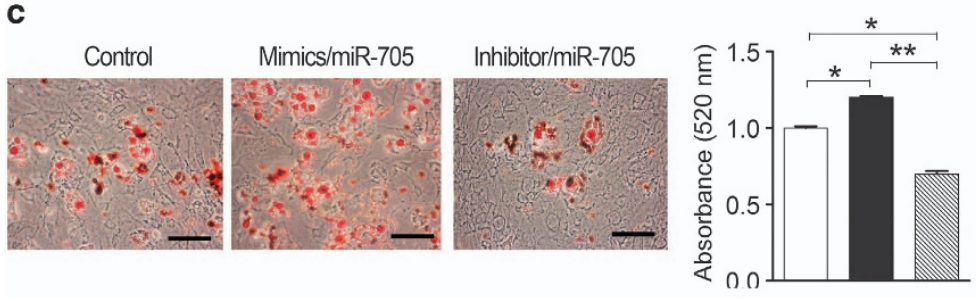

e
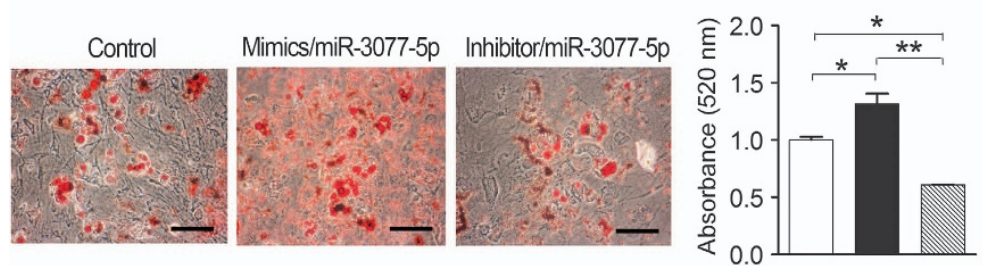
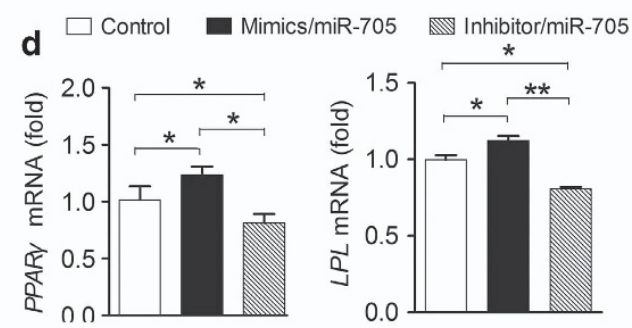

Figure 4 miR-705 and miR-3077-5p promote adipocyte differentiation of MSCs. (a and $\mathbf{b})$ Real-time RT-PCR was used to determine the expression level of miR-705 and miR-3077-5p during different time point of adipogenic induction. (c and d) miR-705 promoted adipocyte differentiation of MSCs. miR-705 mimics, inhibitors and negative controls were transfected into MSCs and induced by adipogenic medium for 7 days. Oil red 0 staining was performed and quantified (c). The representative microscopic view at a magnification of $\times 200$ of cells after staining was shown. The expression of PPAR $\gamma$ and LPL mRNA were measured by real-time RT-PCR (d). (e and f) miR-3077-5p promoted adipocyte differentiation of MSCs. miR-3077-5p mimics, inhibitors and negative controls were transfected into MSCs and induced for 7 days. Oil red 0 staining was performed and quantified (e). The expression of $P P A R \gamma$ and $L P L$ mRNA were measured by real-time RT-PCR (f). Data are shown as means $\pm S . D$. ${ }^{*} P<0.05,{ }^{* *} P<0.01$, $n=3$. Scale bar in micrographs represents $50 \mu \mathrm{m}$

PPAR $\gamma$ and LPL were upregulated by overexpression of miR705 or miR-3077-5p, while they were downregulated by the knockdown of each miRNAs (Figures $4 d$ and $\mathrm{f}$ ).

miR-705 and miR-3077-5p target distinct transcription factors to control MSCs lineage commitment. miRNAs function by targeting the $3^{\prime}$ untranslated region ( $\left.3^{\prime} U T R\right)$ of mRNA with base pair complementarity. ${ }^{21}$ Based on computational miRNA target prediction analysis with several databases (DIANAMT, miRanda, miRwalk, PITA and RNA22), we found that HOXA10 was a predicted target of miR-705, and RUNX2 was a target of miR-3077-5p.

To investigate whether HOXA10 and RUNX2 are regulated post-transcriptionally, we compared their mRNA and protein levels in OVX and Sham MSCs. Real-time RT-PCR showed that HOXA10 mRNA level was slightly reduced (Figure 5a), and Runx2 mRNA level was not changed in OVX MSCs (Figure 5b). In contrast, the protein levels of HOXA10 and Runx2 were significantly decreased (Figure 5c).

In order to confirm miR-705 control HOXA10 expression, we performed gain or loss of function assay of miR-705. Western blot results showed that overexpression of miR-705 reduced the HOXA10 protein level, while knockdown of miR-
705 enhanced its expression in MSCs (Figure 5d). To further determine whether miR-705 directly targets the $3^{\prime} U T R$ of HOXA10 mRNA, we generated luciferase reporter in which the $3^{\prime}$ UTR of HOXA10 was inserted into the multiple cloning site of the pMIR-Report vector. Consistent with bioinformatical prediction, cotransfection of miR-705 mimics downregulated pMIR-HOXA10 reporter activity, while cotransfection of miR705 inhibitor upregulated the reporter activity (Figure 5e).

To confirm that miR-3077-5p regulates RUNX2 expression at the post-transcriptional level, we performed similar assay to miR-3077-5p. Consistently, our results showed that overexpression of miR-3077-5p reduced the protein level of RUNX2 and pMIR-RUNX2 reporter activity, while knockdown of miR-3077-5p increased the protein level of RUNX2 and pMIR-RUNX2 reporter activity (Figures $5 f$ and $g$ ).

Knockdown of miR-705 and miR-3077-5p rescue the cell lineage commitment disorder of MSCs from osteoporosis bone marrow. To further confirm the role of miR-705 and miR-3077-5p in osteoporosis, we tested whether their knockdown in OVX MSCs rescued the osteogenic differentiation disorders. The results showed that separate knockdown of miR-705 or miR-3077-5p recovered the mineralized 


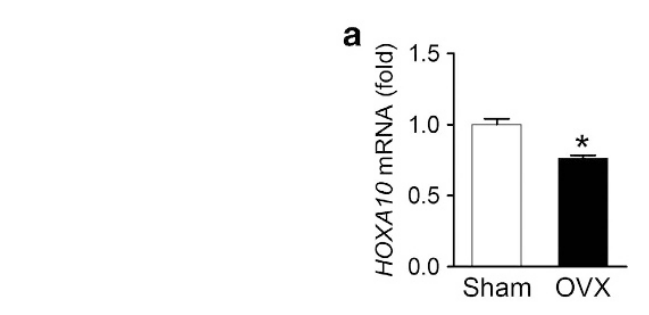

b
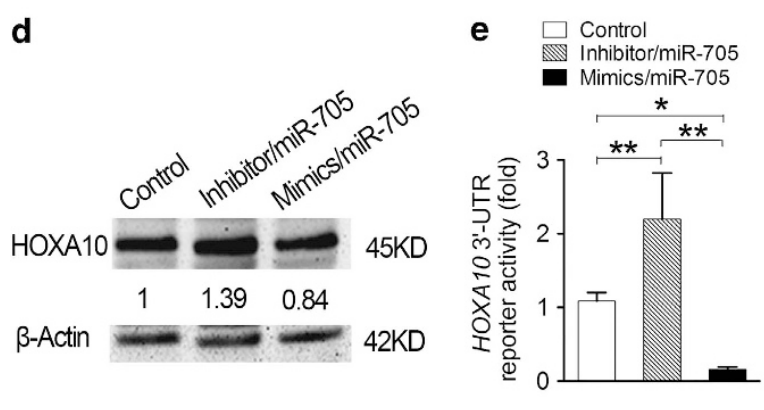

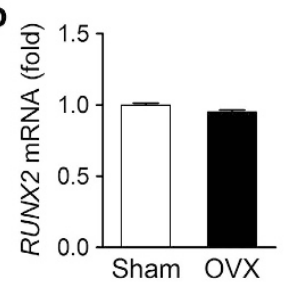

c

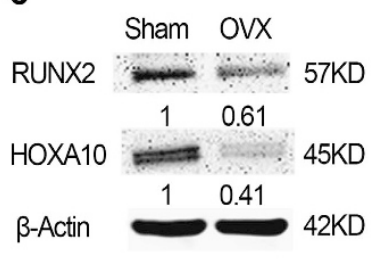

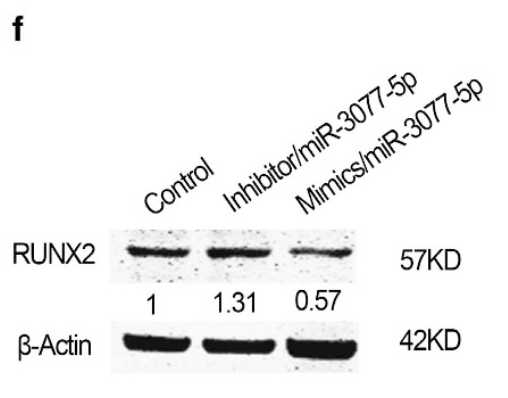

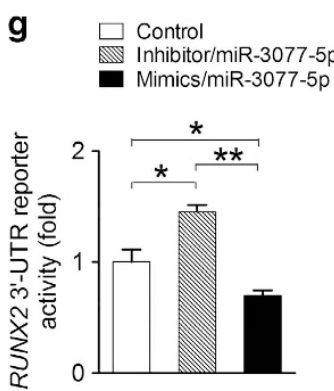

Figure 5 miR-705 and miR-3077-5p bind to the 3'UTR of the HOXA10 and RUNX2 mRNA separately. (a and $\mathbf{b})$ HOXA10 and RUNX2 mRNA level in Sham and OVX MSCs were measured by real-time RT-PCR. Data are shown as means \pm S.D. ${ }^{*} P<0.05$, versus Sham, $n=3$. (c) Western blot was performed to determine the protein level of HOXA10 and RUNX2. (d) Western blot was performed to measure HOXA10 protein level of MSCs after transfected with negative control, miR-705 inhibitor and mimics for $48 \mathrm{~h}$. (e) HOXA10 3'UTR reporter was co-transfected with negative control, miR-705 inhibitor and mimics into MC3T3 cells. Forty-eight hours after transfection, luciferase activities were measured. (f) Western blot was performed to measure HOXA10 protein level of MSCs after transfected with negative control, miR-3077-5P inhibitor and mimics for $48 \mathrm{~h}$. (g) RUNX2 3'UTR reporter was co-transfected with negative control, miR-3077-5P inhibitor and mimics into MC3T3 cells. Forty-eight hours after transfection, luciferase activities were measured. Data are shown as means \pm S.D. ${ }^{\star} P<0.05,{ }^{\star \star} P<0.01, n=4$. Sham, sham surgery; OVX, ovariectomy

node formation of OVX MSCs to a certain extent (Figures 6a and c). Notably, western blot revealed that the knockdown of miR-705 partly recovered HOXA10 protein level (Figure 6b), and knockdown of miR-3077-5p partly recovered RUNX2 protein level during osteogenic differentiation of OVX MSCs (Figure 6d), suggesting that the knockdown of the miRNAs functioned through their target genes. As separate knockdown of miR-705 or miR-3077-5p only partly rescued the osteogenic differentiation of OVX MSCs, we tested whether combined knockdown of miR-705 and miR-3077-5p fully rescued the osteogenic differentiation disorder. Our results showed that combined knockdown of miR-705 and miR3077-5p recovered the mineralization and osteoblast differentiation of OVX MSCs to nearly the level of Sham MSCs (Figures $6 e$ and f).

Then, we tested whether knockdown of miR-705 and miR3077-5p reduces the excessive adipogenic differentiation of OVX MSCs. Oil red O staining results showed that knockdown of miR-705 or miR-3077-5p partly downregulated the adipocyte formation of OVX MSCs (Figures 7a and c). Consistently, HOXA10 protein level was upregulated by miR-705 inhibitor (Figure 7b), and the RUNX2 protein level was upregulated by miR-3077-5p inhibitor in OVX MSCs during adipogenic differentiation (Figure 7d). Moreover, combined knockdown of miR-705 and miR-3077-5p reduced the adipogenic differentiation of OVX MSCs to the general level of Sham MSCs (Figures 7e and f).

TNF $\alpha$ and ROS mediate the overexpression of miR-705 and miR-3077-5p through NF- $\kappa$ B pathway. To investigate the mechanism that enhances miR-705 and miR-3077-5p expression levels during osteoporosis, we tested the direct effect of estrogen on cultured MSCs in vitro. Surprisingly, estrogen significantly upregulated the expression of both miR-705 and miR-3077-5p in vitro (Figure 8a). As estrogen deficiency enhanced miR-705 and miR-3077-5p expression and estrogen injection reduced their expression in vivo (Figures $2 \mathrm{~b}$ and $\mathrm{d}$ ), we speculated that estrogen regulates these miRNAs expression through an indirect way. A number of research showed that inflammatory factors such as tumor necrosis factor alpha (TNF $\alpha$ ) and interferon gamma (IFN $\gamma$ ) secreted by $T$ cells after estrogen deficiency are crucial for the inhibition of bone formation. Meanwhile, accumulated ROS caused by estrogen deficiency were reported to impair osteogenic differentiation of MSCs and osteoblasts. ${ }^{22,23} \mathrm{We}$ confirmed that both levels of TNF $\alpha$ and ROS were elevated in OVX MSCs (data not shown). Then, we tested whether TNF $\alpha$ and ROS regulate the miRNAs expression. Our results showed both TNF $\alpha$ and hydrogen peroxide $\left(\mathrm{H}_{2} \mathrm{O}_{2}\right.$, the dominant component of intracellular ROS) significantly upregulated the expression of miR-705 and miR-3077-5p (Figures $8 \mathrm{c}$ and $\mathrm{d}$ ). To investigate the mechanism of their regulation, we analyzed the promoter regions of pri-miR-705 and pri-miR-3077-5p, and found that there were several binding sites for NF- $\kappa \mathrm{B} 1$ and NF- $\kappa \mathrm{B} 2$ on both promoters. Western blot showed the NF- $\kappa$ B pathway was activated in OVX MSCs (Figure 8b). Then, to confirm its effect on miRNAs expression, we inhibited NF- $\kappa$ B pathway by knockdown of IKK $\alpha$ with SiRNA (Supplementary Figure S5A, S5B). The results showed that knockdown of IKK $\alpha$ significantly reduced the expression of miR-705 and miR-3077-5p (Figure 8e). Notably, knockdown of IKK $\alpha$ arrested the elevation of both miRNAs led by TNF $\alpha$ and $\mathrm{H}_{2} \mathrm{O}_{2}$ (Figures $8 f$ and $g$ ). 

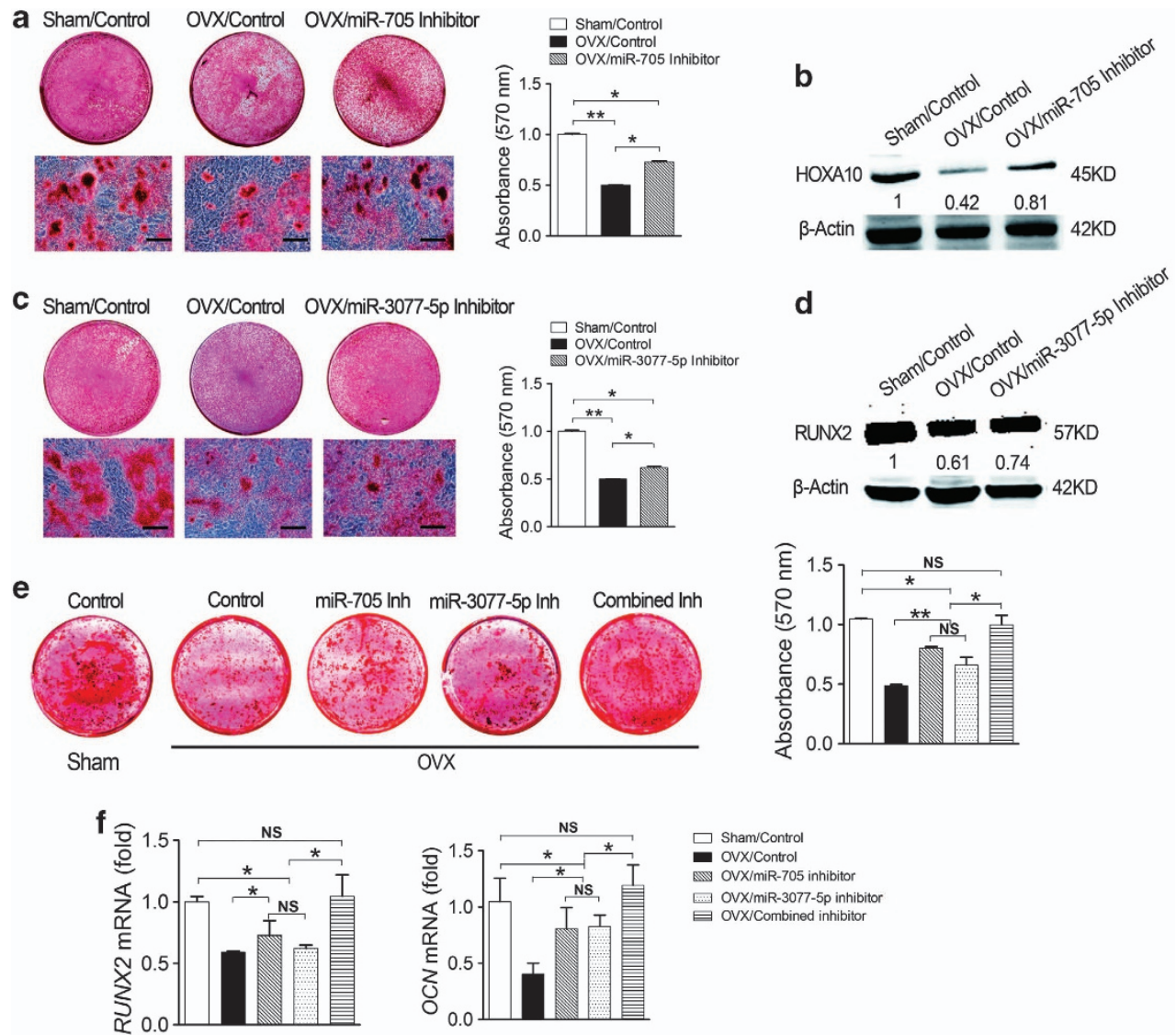

Figure 6 Knockdown of miR-705 and miR-3077-5p promote osteogenic differentiation of OVX MSCs. (a and b) miR-705 inhibitor promoted osteoblast differentiation of OVX MSCs. Sham and OVX MSCs were transfected with negative control and miR-705 inhibitor, and cultured with osteogenic medium for 14 days. Alizarin red S staining was performed and quantified (a). The representative microscopic view at a magnification of $\times 100$ of cells after staining was shown. Scale bar in micrographs represents $100 \mu \mathrm{m}$. The expression of HOXA10 protein was measured by western blot (b). (c and d) miR-3077-5P inhibitor promoted osteoblast differentiation of OVX MSCs through upregulating RUNX2 protein level. Alizarin red S staining was performed and quantified 14 days after osteogenic induction (c). The expression of HOXA10 protein was measured by western blot (d). (e and f) Inhibitor of miR-705 and miR-3077-5p were combined together and transfected into OVX MSCs. Fourteen days after osteogenic induction, the alizarin red staining was performed (e) and the expression of $R U N X 2$ and $O C N$ in MSCs were measured by real-time RT-PCR (f). Data are shown as means $\pm S . D$. ${ }^{*} P<0.05$, ${ }^{* *} P<0.01, n=3$. Sham, sham surgery; OVX, ovariectomy; Inh, inhibitor

\section{Discussion}

This study that identified miR-705 and miR-3077-5p were novel negative regulators of osteoblast differentiation and positive regulators of adipogenic differentiation of MSCs at the post-transcriptional level. Furthermore, our results suggested the overexpression of both miRNAs mediated the shift of mesenchymal stem cell lineage commitment to adipocyte in osteoporosis bone marrow.

A subset of miRNAs, such as miR-34s, miR-335, miR-26a, miR-204/211 and so on, were reported to regulate MSCs lineage commitment and have a crucial role in bone development. ${ }^{17,24,25}$ However, whether miRNAs are related with bone diseases such as osteoporosis is largely unknown. Based on comprehensive miRNAs profiling, we found miR705 and miR-3077-5p overexpressed in MSCs from osteoporosis bone marrow. Moreover, consistent with the recovery of osteoporosis, in-vivo estrogen treatment significantly reduced the excessive miR-705 and miR-3077-5p in OVX mice. The positive correlation between the level of these miRNAs and the severity of osteoporosis suggested miR-705 and miR-3077-5p have a crucial role in the etiology of osteoporosis.

As miR-705 and miR-3077-5p were not discovered until recently by miRNA sequencing technology, there was no report about their biological function. As many miRNAs that have unique tissue-specific expression patterns were reported to be crucial to the development or function of specific tissue, we investigated the expression profile of miR705 and miR-3077-5p in various tissues. Both miRNAs were preferentially expressed in the bone, which suggested that they may have an important role in its development and homeostasis. To further investigate whether miR-705 and miR-3077-5p regulate the differentiation of MSCs, we profiled their expression levels at different time points during both osteoblast and adipogenic differentiation of MSCs. Interestingly, both miRNAs were downregulated during osteoblast differentiation, while, oppositely, upregulated during adipogenic differentiation. The following gain or loss of function assay demonstrated both miRNAs could inhibit MSCs osteoblast differentiation and promote adipogenic differentiation. Taken together, our results suggested miR-705 and 


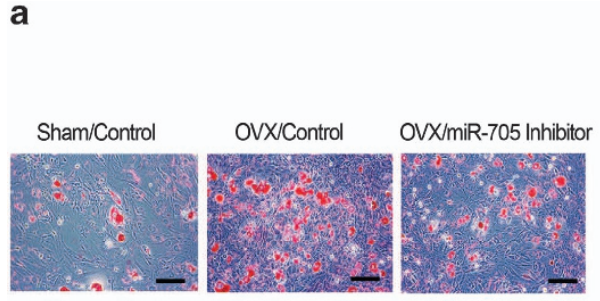

C

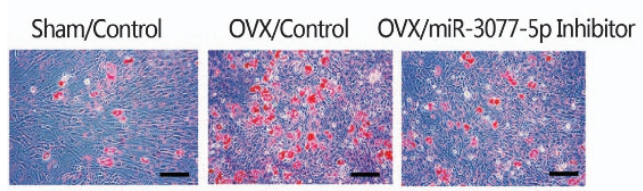

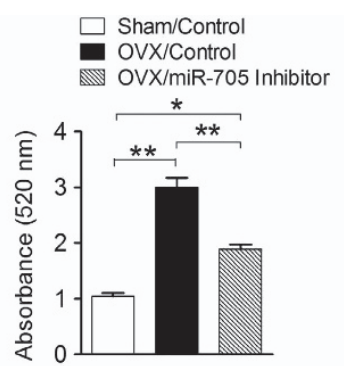
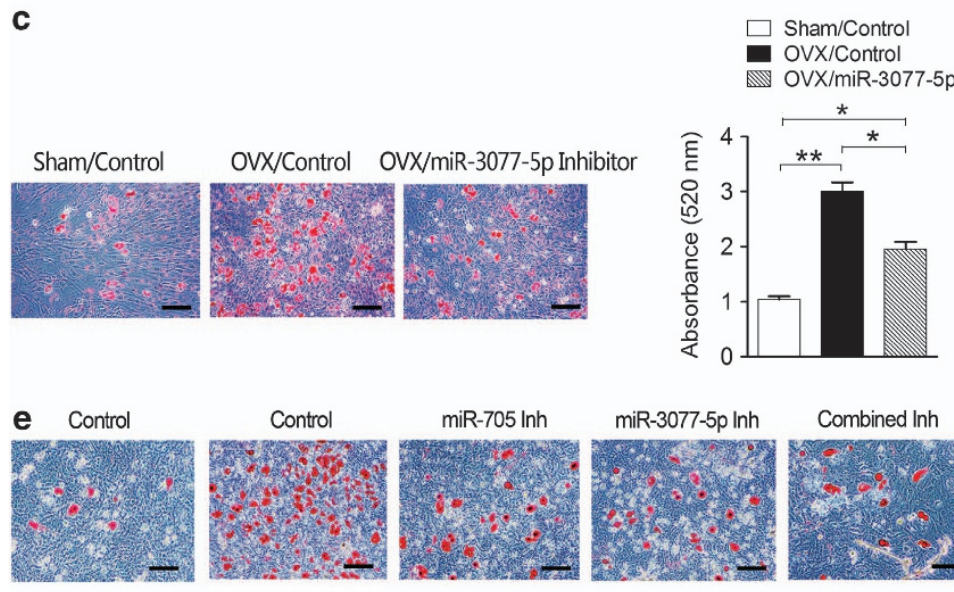

miR-705 Inh
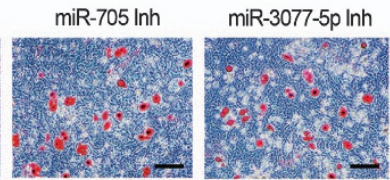

Combined Inh

Sham

OVX
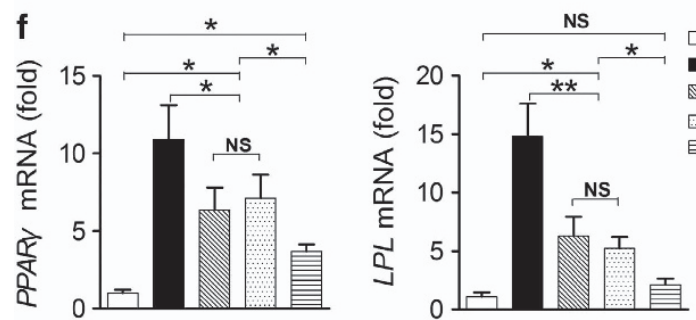
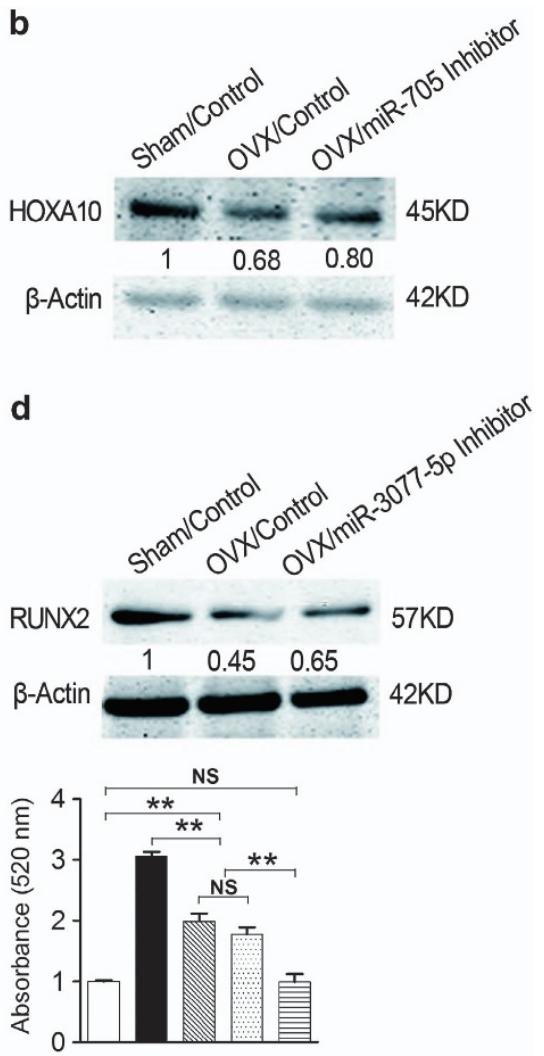

Figure 7 Knockdown of miR-705 and miR-3077-5p inhibit adipogenic differentiation of OVX MSCs. (a and $\mathbf{b})$ miR-705 inhibitor reduced adipocyte differentiation of OVX MSCs through upregulating HOXA10 protein level. Sham and OVX MSCs were transfected with negative control and miR-3077-5p inhibitor, and cultured with adipogenic medium for 7 days. Oil red $O$ staining was performed and quantified (a). The representative microscopic view at a magnification of $\times 200$ of cells after staining was shown. Scale bar in micrographs represents $50 \mu \mathrm{m}$. The expression of HOXA10 protein was measured by western blot (b). (c and d) miR-3077-5P inhibitor reduced adipocyte differentiation of OVX MSCs. Oil red O staining was performed and quantified 7 days after adipocyte induction (c). The expression of HOXA10 protein was measured by western blot (d). (e and f) Inhibitor of miR-705 and miR-3077-5p were combined together and transfected into OVX MSCs. Seven days after adipogenic induction, the oil red 0 staining was performed (e) and the expression of $P P A R \gamma$ and $L P L$ in MSCs were measured by real-time RT-PCR (f). Data are shown as means \pm S.D. ${ }^{*} P<0.05,{ }^{* *} P<0.01$, $n=3$. Sham, sham surgery; OVX, ovariectomy; Inh, inhibitor

miR-3077-5p are both novel regulators of cell lineage commitment of MSCs.

As miRNAs function by targeting the $3^{\prime}$ untranslated region (3'UTR) of mRNA with base-pair complementarity, ${ }^{21}$ bioinformatical analysis was reported to be a powerful method to predict the target gene. ${ }^{26}$ With computational miRNA target prediction analyses, we found that HOXA10 was a predicted target of miR-705, and RUNX2 was a target of miR-3077-5p. According to reports, Runx2 is the master regulator of the osteogenic differentiation. ${ }^{27} \mathrm{HOXA10}$, a member of the family of homeodomain-containing transcription factors, acts as both a stimulator of Runx2 and a suppressor of PPAR $\gamma$ in stromal cell differentiation. ${ }^{28,29}$ Functional verification showed that RUNX2 or HOXA10 deficiency led to osteoblast differentiation disorder and serious skeleton developmental defects, while their overexpression inhibited adipocyte differentiation of MSCs. ${ }^{25,30,31}$ With gain or loss of function assay and luciferase reporter assay, we showed miR-705 could directly target the HOXA10 mRNA 3' UTR, while miR-3077-5p could directly target the RUNX2 mRNA 3'UTR. Moreover, although the mRNA level of RUNX2 and HOXA10 were not obviously changed in MSCs from osteoporosis bone marrow, we found their protein level to be significantly downregulated, suggesting that the post-transcriptional regulation by miRNAs had a crucial role in the shift of cell lineage commitment in MSCs during osteoporosis. Several lines of evidence indicated that miR-705 and miR-3077-5p worked together to mediate the shift of cell lineage commitment of MSCs to adipocytes during 


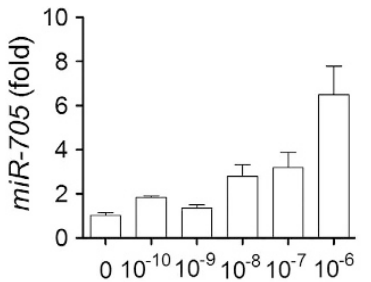

C

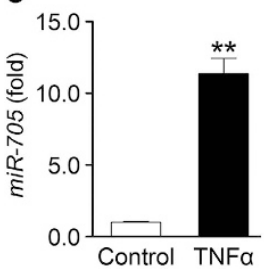

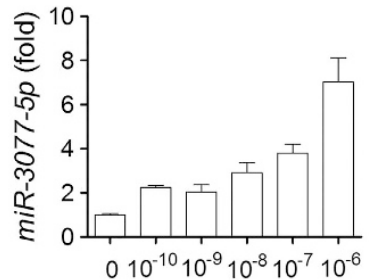

d

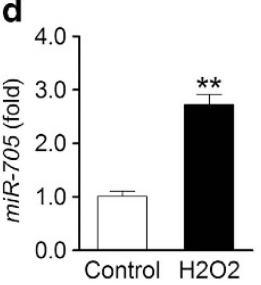

\section{$\square$ Control}

- TNFa

TNFa +IKKa SiRNA

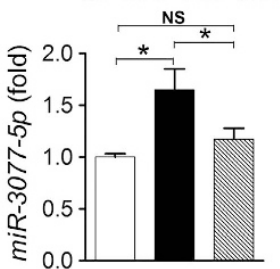

b
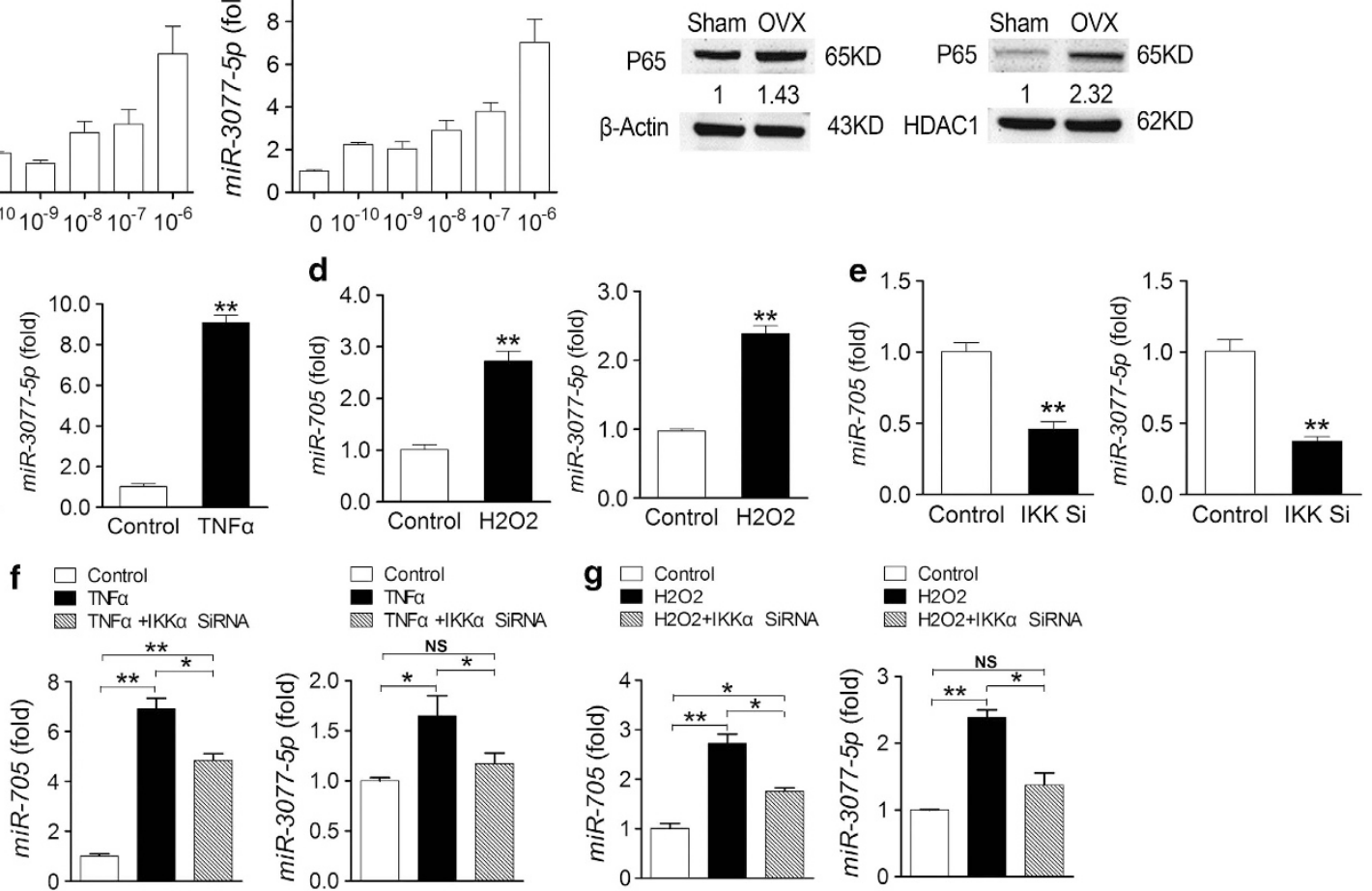

Figure $8 \mathrm{TNF} \alpha$ and $\mathrm{H} 2 \mathrm{O} 2$ upregulated miR-705 and miR-3077-5p expression through NF- $\kappa$ B pathway. (a) MSCs was cultured with different concentration of estrogen (0, $10^{-10}, 10^{-9}, 10^{-8}, 10^{-7}$ and $10^{-6} \mathrm{M}$ ) for $72 \mathrm{~h}$. The expression of miR-705 and miR-3077-5p were measured by real-time RT-PCR. (b) Western blot was performed to detect the level of P65 in cytoplasm and nucleus of OVX and Sham MSCs. $\beta$-actin was used as the control for cytoplasmic P65 and HDAC1 was used as the control for P65 in nucleus. (c) MSCs was cultured in culture medium supplemented with $10 \mathrm{ng} / \mathrm{ml} \mathrm{TNF} \alpha$ for $72 \mathrm{~h}$. Real-time RT-PCR was performed to detect the expression of miR-705 and miR-3077-5p. (d) Real-time RT-PCR was performed to detect the expression of miR-705 and miR-3077-5p after being stimulated with $10^{-5} \mathrm{M} \mathrm{H}_{2} \mathrm{O}_{2}$ for $72 \mathrm{~h}$. (e) The MSCs was transfected with IKK $\alpha$ SiRNA for $48 \mathrm{~h}$ and real-time RT-PCR was performed. (f) The MSCs was transfected with negative control and IKK $\alpha$ SiRNA. Forty-eight hours after transfection, MSCs was cultured in culture medium supplemented with $10 \mathrm{ng} / \mathrm{ml}$ TNF $\alpha$ for $72 \mathrm{~h}$. Real-time RT-PCR was performed to detect the expression of miR-705 and miR-3077-5p. (g) MSCs was cultured in culture medium supplemented with $10^{-5} \mathrm{M} \mathrm{H} 2 \mathrm{O} 2$ for $72 \mathrm{~h}$ after IKK $\alpha$ SiRNA transfection and real-time RT-PCR was performed. Data are shown as means \pm S.D. ${ }^{*} P<0.05,{ }^{* \star} P<0.01, n=3$. Sham, sham surgery; OVX, ovariectomy; Si, IKK $\alpha$ SiRNA

osteoporosis. Firstly, the expression pattern of both miRNAs were similar during osteoporosis. Secondly, both miRNAs functioned as negative regulators of osteoblast differentiation and positive regulators of adipogenic differentiation of MSCs. Thirdly, their target genes, RUNX2 and HOXA10, are involved in the bone morphogenetic proteins (BMPs) signaling pathway, ${ }^{32}$ providing synergistic effect to promote osteoblast differentiation of MSCs. ${ }^{29}$ Fourthly, knockdown of either miRNAs alone could only partly rescued the differentiation disorder of MSCs from osteoporosis bone marrow, while combined knockdown of mir-705 and mir-3077-5p mainly rescued the cell lineage commitment disorder. Taken together, our findings suggested that different miRNAs are involved in a complicated network to regulate the differentiation of MSCs in both physiological and pathological conditions.

To investigate the mechanism that controls the synergistic role of miR-705 and miR-3077-5p after estrogen deficiency, we compared the promoter region of pri-miR-705 and pri-miR$3077-5 p$ and found that NF- $\kappa$ B could combine on both their promoters. A number of research reported that estrogen deficiency indirectly led to osteoporosis through enhancing TNF $\alpha$ secretion and intracellular ROS accumulation. ${ }^{22,23} \mathrm{We}$ showed that, redundant TNF $\alpha$ and ROS in OVX mice enhanced the levels of miR-705 and miR-3077-5p through activating NF- $\kappa$ B pathway. Although $\mathrm{TNF} \alpha$ and ROS were reported to inhibit osteoblast differentiation, none of these reports showed that TNF $\alpha$ and ROS, to a certain extent, function through regulating the expression of specific miRNAs. Our findings suggested a new approach of TNF $\alpha$ and ROS to inhibit MSCs differentiation and cause osteoporosis after menopause and ageing.

Anabolic drugs, such as PTH and vitamin D, have been used in clinics to stimulate bone formation and treat osteoporosis. ${ }^{33}$ However, long-term trials showed that these drugs had no effects on the prevention of hip fracture. The side-effects, such as hypercalcaemia, nausea, diarrhea, also affect long-term administration and adherence. ${ }^{33} \mathrm{New}$ anabolic drugs are extremely necessary to rebuild strong bones. With new strategies, restoration of silenced miRNAs or suppression of overexpressed miRNAs are available in vivo. ${ }^{34}$ Successful in-vivo studies on cancer supported the notion that miRNAs could be used as innovative therapeutics to address unmet needs. ${ }^{34}$ Here, we have shown that suppression of elevated miRNAs by synthetic oligonucleotides rescued the shift of cell lineage commitment of OVX MSCs, suggesting miRNAs as the potential therapeutic target for the treatment of osteoporosis as well as stem cell-mediated regenerative medicine. 


\section{Materials and Methods}

Animals. All animal procedures were performed according to the guidelines of the Animal Care Committee of the Fourth Military Medical University, X'ian, China. Sixty-six-week-old female C57BL/6J mice were randomly divided into two groups, and they underwent either sham surgery or bilateral ovariectomy (OVX) under general anesthesia by the dorsal approach. Then all mice were housed under specific pathogen-free conditions $\left(22^{\circ} \mathrm{C}\right.$, 12-hour light/12-hour dark cycles and $50-55 \%$ humidity) with free access to food pellets and tap water for 3 months.

Cell culture. Three months after OVX and sham surgery, the mouse MSCs in hind limbs were isolated and cultured as previously described. ${ }^{35}$ Briefly, after euthanasia, the hind limbs were aseptically removed and bones were dissected free of soft tissues. Marrow cavities of both femur and tibia were flushed with growth medium containing $\alpha$-MEM (Invitrogen, Carlsbad, CA, USA) supplemented with $10 \%$ FBS, $1 \%$ penicillin and streptomycin. The cell suspension was seeded in $10-\mathrm{cm}$ tissue culture dishes and grown in the growth medium in a humidified atmosphere of $5 \% \mathrm{CO} 2$ at $37^{\circ} \mathrm{C}$. The medium was changed every 2-3 days to remove non-adherent cells, and adherent cells were cultured until they were confluent. At confluence, BMSCs were passaged after digestion with $0.25 \%$ trypsin/1 mM EDTA.

Alizarin red staining and oil-red staining. To induce MSC osteoblast differentiation, we grew MSCs in osteogenesis-inducing media containing $100 \mu \mathrm{g} /$ $\mathrm{ml}$ ascorbic acid, $2 \mathrm{mM} \beta$-glycerophosphate and $10 \mathrm{nM}$ dexamethasone. Alizarin red staining was performed as described previously. ${ }^{36}$ To induce adipogenic differentiation, we cultured MSCs in adipogenesis-inducing media containing $0.5 \mathrm{mM}$ isobutylmethylxanthine, $0.5 \mu \mathrm{M}$ dexamethasone and $60 \mu \mathrm{M}$ indomethacin (Sigma-Aldrich, St. Louis, MO, USA). The media were changed every 3 days. After 3 weeks of culture in vitro, Oil Red $O$ staining was performed to detect the lipid droplets as described previously. ${ }^{37}$

Histomorphometric analysis of bone marrow fat. Bone sections were stained with toluidine blue stain. Mineralized bone tissue was stained blue, whereas the unstained area in the bone marrow represented empty spaces previously occupied by adipocytes. Photos were taken under magnification of $\times 20$ on five consecutive microscopic fields of the secondary spongiosa of the distal femur. Fat cells identified as empty oval spaces in the photos were measured with Image-Pro software (Media Cybernetics, Rockville, MD, USA). Static measurements were performed on three individual bone samples per group and fat volume and adipocyte size per high-power field were calculated.

miRNAs microarray and data processing. MiRNAs microarray and data processing were performed by LC Sciences (Houston, TX, USA). In brief, Total RNA $(20 \mu \mathrm{g})$ was extracted by TRIzol method (Invitrogen) when cultured MSCs reached $80 \%$ confluence. RNA of MSCs derived from three mice were mixed as one sample for microarray detection. The miRNAs microarray was performed using the LC Sciences microarray platform (LC Sciences). The platform contained miRNA probe region detecting the miRNA transcripts listed in the Sanger miRNA database v17.0 (http://www.mirbase.org/). Multiple and TMnormalized control probes were included on each chip. Hybridization was detected by fluorescence labeling with tag-specific $\mathrm{Cy} 3$ and $\mathrm{Cy} 5$ dyes. Hybridization images were collected using a laser scanner (GenePix 4000B, Molecular Device, Sunnyvale, CA, USA) and digitized using Array-Pro image analysis software (Media Cybernetics). Data were analyzed by first subtracting the background and then normalizing the signals using a LOWESS filter (locally-weighted regression). Detectable signals with average intensity three-times higher than background S.D. and CV (S.D./average intensity) $<0.5$ were included in further analysis. The images were displayed in pseudo colors to expand the dynamic visual range. For the Cy3 and Cy5 images, as the signal intensity increased from 1 to 65535 , the corresponding color changed from blue to green to yellow to red. The miRNA with signal intensity $>32$ was considered as detectable and signal intensity $>500$ was considered as highly expressed by the system. The signals were processed and compared by calculating their ratio (log2-transformed, balanced). The data of three independent experiments were used for $t$-test.

Real-time RT-PCR of mRNA and microRNA. Total RNA was isolated using Trizol reagent (Invitrogen) according to the manufacturer's standard instructions. For reverse transcription of mRNA, random-primed cDNA was synthesized from $2 \mu \mathrm{g}$ of total RNA using a PrimeScript RT reagent kit (TaKaRa, Dalian, China). For RT-PCR of microRNAs, miRNA was reverse-transcribed using specific RT primers (RiboBio, Guangzhou, China). Real-time PCR analysis was performed using the SYBR Premix Ex Taq II kit (TaKaRa) and detected on the ABI Prism $7500 \mathrm{HT}$ sequence detection system (Applied Biosystems, Foster City, CA, USA). $\beta$-actin and U6 were used as loading controls for quantitation of mRNA and miRNAs. The Bulge-Loop miRNA qRT-PCR Primer Set (RiboBio) were used for real-time qRT-PCR of miR-705 (Product ID: miRQ0003495-1-2), miR-3077-5p (Product ID: miRQ0014862-1-2) and U6 (Product ID: MQP-0202). The primers for protein-encoding genes were listed in the Supplementary Table S1.

Estrogen treatment in vivo. Two months after the ovariectomy, the mice were injected intraperitoneally with $50 \mu \mathrm{g} / \mathrm{kg}$ body weight of $17 \beta$ estradiol (SigmaAldrich, Cat No. E2257) or sesame oil (as a vehicle control) every other day for 4 weeks.

Transfection of miRNA mimics and inhibitors. The miRNA mimics, inhibitors and negative controls of miR-705 and miR-3077-5p were purchased from RiboBio. The cells were transfected with a mimic, an inhibitor and a negative control at final concentrations of $50 \mathrm{nM}$. The siPORT NeoFX transfection agent (Ambion, Austin, TX, USA) was used according to the manufacturer's instructions. In brief, the cells were digested with $0.25 \%$ trypsin when they reached $80 \%$ confluence. Diluted miRNA mimics/inhibitors and diluted siPORT NeoFX Transfection Agent were mixed and incubated for $10 \mathrm{~min}$ at room temperature. Cell suspensions were overlaid onto the transfection complexes and incubated at $37^{\circ} \mathrm{C}$ for $48 \mathrm{~h}$ until further assay.

Luciferase reporter assay. Target gene $3^{\prime} U T R$ reporter was constructed and transfected as described previously. ${ }^{20}$ The HOXA10 oligonucleotide sequences were amplified using primers (forward: $5^{\prime}$-GGACTAGTCTGAGCG CCTGGACCCAT-3'; reverse: 5'-CCCAAGCTTTTAAACACAGCCCAGCACTCC$3^{\prime}$ ) with HindllI and Spel sites at their extremities to insert the pMIR-Report vector (Ambion, Austin, TX, USA). RUNX2 oligonucleotide sequences were amplified using primers (forward: $5^{\prime}$-GGACTAGTGCCAATCCCAGCATTCCT-3'; reverse: $5^{\prime}$ CGACGCGTTGGTGTGCTTCAAGCTAC- $3^{\prime}$ ). Before transfection, cells were transplanted in $96-$ well plates at $70 \%$ confluence. All transfections were conducted using Lipofectamine 2000 (Invitrogen). The pMIR-Report (pMIR-Cont), pMIRHOXA10, pMIR-RUNX2 and pMIR- $\beta$-gal plasmids were used as reporter constructs and were cotransfected into cells with a miRNA mimics or inhibitor. After $48 \mathrm{~h}$, firefly luciferase activity (Luciferase Assay System, Promega, Madison, WI, USA) and $\beta$-gal activity (b-galactosidase Assay Kit, Beyotime, ShangHai, China) were determined in cell lysates according to the manufacturers' recommended protocols. To control the transfection efficiency, luciferase activity was normalized to that of $\beta$-gal.

Western blot analysis. The western blot analysis were performed as previously described. ${ }^{20}$ Whole-cell lysates were extracted with lysis buffer for western blotting. The proteins were loaded on $10 \%$ sodium dodecyl sulfatepolyacrylamide gels, transferred to polyvinylidene fluoride membranes (Millipore, Billerica, MA, USA) and blocked with 5\% nonfat milk powder in PBST (PBS with $0.1 \%$ Tween). The membranes were probed overnight with the following primary antibodies. The primary antibody for mouse RUNX2(Abcam, Cambridge, MA, USA), $\beta$-actin(Cell Signaling, Beverly, MA, USA), HOXA10(Santa Cruz, Dallas, TX, USA), P65(Cell Signaling) and HDAC1 (Cell Signaling) were used in this study. Then, the membranes were incubated with peroxidase-conjugated secondary antibody (Boster, Wuhan, China). The blots were visualized using an enhanced chemiluminescence kit (Amersham Biosciences, Piscataway, NJ, USA) according to the manufacturer's recommended instructions. The gray value of the blots in the pictures were measured with IMAGE J software (http://rsb.info.nih.gov/ij/) by following the instructions. The gray value of each target protein was normalized to that of $\beta$-actin before comparison.

Transfection of IKK $\alpha$ SiRNA. The IKK $\alpha$ SiRNA were purchased from RiboBio. The cells were transfected with IKK $\alpha$ SiRNA and negative control at final concentrations of $100 \mathrm{nM}$. The Lipofectamine 2000 (Invitrogen) was used according to the manufacturer's instructions. In brief, cells were passaged in six-well plates, and were $30-50 \%$ confluent at the time of transfection. The diluted oligomer was combined with the diluted Lipofectamine 2000 and added to each well. The cells were incubated at $37^{\circ} \mathrm{C}$ in the incubator for $48 \mathrm{~h}$ before further assay. 
Statistics. Data are presented as mean \pm S.D. Comparisons were made using a one-way ANOVA. All experiments were repeated at least three times, and representative experiments were shown. Differences were considered significant at $P<0.05$

\section{Conflict of Interest}

The authors declare no conflict of interest.

Acknowledgements. This work was supported by grants from the National Major Scientific Research Program of China (2010CB944800 and 2011CB964700) and the Nature Science Foundation of China $(81020108019$, 31030033).

\section{Author Contributions}

Li Liao and Xiaohong Yang: conception and design, collection and assembly of data, data analysis and interpretation and manuscript writing; both authors contributed equally to this work; Xiaoxia Su: collection and/or assembly of data, data analysis and interpretation; Chenghu $\mathrm{Hu}$ : conception and design, data analysis and interpretation; Xiaofei Zhu: collection and/or assembly of data; Nan Yang: collection and/or assembly of data, provision of study material or patients; Xiaoyan Chen: collection and/or assembly of data; Songtao Shi: conception and design; Shenggen Shi: data analysis and interpretation, administrative support; Yan Jin: conception and design, data analysis and interpretation, manuscript writing and financial support of manuscript.

1. Verma S, Rajaratnam JH, Denton J, Hoyland JA, Byers RJ. Adipocytic proportion of bone marrow is inversely related to bone formation in osteoporosis. J Clin Pathol 2002; 55: 693-698.

2. Chan GK, Duque G. Age-related bone loss: old bone, new facts. Gerontology 2002; 48: 62-71.

3. Kirkland JL, Tchkonia T, Pirtskhalava T, Han J, Karagiannides I. Adipogenesis and aging: does aging make fat go MAD? Exp Gerontol 2002; 37: 757-767.

4. Justesen J, Stenderup K, Ebbesen EN, Mosekilde L, Steiniche T, Kassem M. Adipocyte tissue volume in bone marrow is increased with aging and in patients with osteoporosis. Biogerontology 2001; 2: 165-171.

5. Rosen CJ, Ackert-Bicknell C, Rodriguez JP, Pino AM. Marrow fat and the bone microenvironment: developmental, functional, and pathological implications. Crit Rev Eukaryot Gene Expr 2009; 19: 109-124.

6. Kassem M, Marie PJ. Senescence-associated intrinsic mechanisms of osteoblast dysfunctions. Aging Cell 2011; 10: 191-197.

7. Nishikawa K, Nakashima T, Takeda S, Isogai M, Hamada M, Kimura A et al. Maf promotes osteoblast differentiation in mice by mediating the age-related switch in mesenchymal cell differentiation. J Clin Invest 2010; 120: 3455-3465.

8. Rodriguez JP, Astudillo P, Rios S, Pino AM. Involvement of adipogenic potential of human bone marrow mesenchymal stem cells (MSCs) in osteoporosis. Curr Stem Cell Res The 2008; 3: 208-218.

9. Rosen CJ. Growth hormone, insulin-like growth factors, and the senescent skeleton: Ponce de Leon's Fountain revisited? J Cell Biochem 1994; 56: 348-356.

10. Feng X, McDonald JM. Disorders of bone remodeling. Annu Rev Pathol 2011; 6: 121-145.

11. Ye L, Fan Z, Yu B, Chang J, Al Hezaimi K, Zhou X et al. Histone demethylases KDM4B and KDM6B promotes osteogenic differentiation of human MSCs. Cell Stem Cell 2012; 11: 50-61.

12. Mendez-Ferrer S, Michurina TV, Ferraro F, Mazloom AR, Macarthur BD, Lira SA et al. Mesenchymal and haematopoietic stem cells form a unique bone marrow niche. Nature 2010; 466: 829-834.

13. Wei Y, Chen YH, Li LY, Lang J, Yeh SP, Shi B et al. CDK1-dependent phosphorylation of EZH2 suppresses methylation of $\mathrm{H} 3 \mathrm{~K} 27$ and promotes osteogenic differentiation of human mesenchymal stem cells. Nat Cell Biol 2011; 13: 87-94.

14. Kawai M, Rosen CJ. PPARgamma: a circadian transcription factor in adipogenesis and osteogenesis. Nat Rev Endocrinol 2010; 6: 629-636.
15. Harada S, Rodan GA. Control of osteoblast function and regulation of bone mass. Nature 2003; 423: 349-355.

16. Carthew RW, Sontheimer EJ. Origins and mechanisms of miRNAs and siRNAs. Cell 2009; 136: $642-655$.

17. Lian JB, Stein GS, van Wijnen AJ, Stein JL, Hassan MQ, Gaur T et al. MicroRNA control of bone formation and homeostasis. Nat Rev Endocrinol 2012; 8: 212-227.

18. Li H, Xie H, Liu W, Hu R, Huang B, Tan YF et al. A novel microRNA targeting HDAC5 regulates osteoblast differentiation in mice and contributes to primary osteoporosis in humans. J Clin Invest 2009; 119: 3666-3677.

19. Yu JM, Wu X, Gimble JM, Guan X, Freitas MA, Bunnell BA. Age-related changes in mesenchymal stem cells derived from rhesus macaque bone marrow. Aging Cell 2011; 10: 66-79.

20. Liu Y, Liu W, Hu C, Xue Z, Wang G, Ding B et al. MiR-17 modulates osteogenic differentiation through a coherent feed-forward loop in mesenchymal stem cells isolated from periodontal ligaments of patients with periodontitis. Stem Cells 2011; 29: 1804-1816.

21. Bartel DP. MicroRNAs: target recognition and regulatory functions. Cell 2009; 136 : 215-233.

22. Weitzmann MN, Pacifici R. Estrogen deficiency and bone loss: an inflammatory tale. J Clin Invest 2006; 116: 1186-1194.

23. Wauquier F, Leotoing L, Coxam V, Guicheux J, Wittrant $Y$. Oxidative stress in bone remodelling and disease. Trends Mol Med 2009; 15: 468-477.

24. Wei J, Shi Y, Zheng L, Zhou B, Inose H, Wang J et al. miR-34s inhibit osteoblast proliferation and differentiation in the mouse by targeting SATB2. J Cell Biol 2012; 197 509-521.

25. Zhang $\mathrm{Y}$, Xie RL, Croce CM, Stein JL, Lian JB, van Wijnen AJ et al. A program of microRNAs controls osteogenic lineage progression by targeting transcription factor Runx2. Proc Natl Acad Sci USA 2011; 108: 9863-9868.

26. Lewis BP, Burge CB, Bartel DP. Conserved seed pairing, often flanked by adenosines, indicates that thousands of human genes are microRNA targets. Cell 2005; 120 : 15-20.

27. Long F. Building strong bones: molecular regulation of the osteoblast lineage. Nat Rev $\mathrm{Mol}$ Cell Biol 2012; 13: 27-38.

28. Hassan MQ, Tare R, Lee SH, Mandeville M, Weiner B, Montecino M et al. HOXA10 controls osteoblastogenesis by directly activating bone regulatory and phenotypic genes. Mol Cell Biol 2007; 27: 3337-3352.

29. Hassan MQ, Saini S, Gordon JA, van Wijnen AJ, Montecino M, Stein JL et al. Molecula switches involving homeodomain proteins, HOXA10 and RUNX2 regulate osteoblastogenesis. Cells Tissues Organs 2009; 189: 122-125.

30. Chen JR, Zhang J, Lazarenko OP, Kang P, Blackburn ML, Ronis MJ et al. Inhibition of fetal bone development through epigenetic down-regulation of HoxA10 in obese rats fed highfat diet. FASEB J 2012; 26: 1131-1141.

31. Huang J, Zhao L, Xing L, Chen D. MicroRNA-204 regulates Runx2 protein expression and mesenchymal progenitor cell differentiation. Stem Cells 2010; 28: 357-364.

32. de la Fuente L, Helms JA. Head, shoulders, knees, and toes. Dev Biol2005; 282: 294-306.

33. Rachner TD, Khosla S, Hofbauer LC. Osteoporosis: now and the future. Lancet 2011; 377: $1276-1287$

34. Kim M, Kasinski AL, Slack FJ. MicroRNA therapeutics in preclinical cancer models. Lancet Oncol 2011; 12: 319-321.

35. Edgar CM, Chakravarthy V, Barnes G, Kakar S, Gerstenfeld LC, Einhorn TA. Autogenous regulation of a network of bone morphogenetic proteins (BMPs) mediates the osteogenic differentiation in murine marrow stromal cells. Bone 2007; 40: 1389-1398.

36. Chang J, Wang Z, Tang E, Fan Z, McCauley L, Franceschi R et al. Inhibition of osteoblastic bone formation by nuclear factor-kappaB. Nat Med 2009; 15: 682-689.

37. Guo W, He Y, Zhang X, Lu W, Wang C, Yu H et al. The use of dentin matrix scaffold and dental follicle cells for dentin regeneration. Biomaterials 2009; 30: 6708-6723.

(1) () (2) Cell Death and Disease is an open-access journal published by Nature Publishing Group. This work is licensed under a Creative Commons Attribution-NonCommercialShareAlike 3.0 Unported License. To view a copy of this license, visit http://creativecommons.org/licenses/by-nc-sa/3.0/

\section{Supplementary Information accompanies this paper on Cell Death and Disease website (http://www.nature.com/cddis)}

